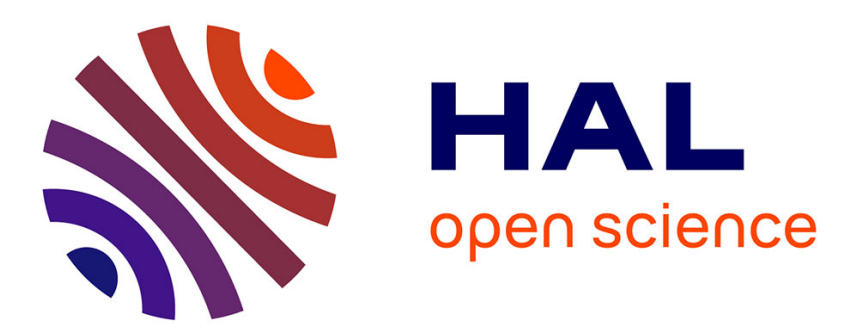

\title{
Strict Lyapunov functions for time-varying systems with persistency of excitation
}

Mohamed Maghenem, Antonio Loria

\section{To cite this version:}

Mohamed Maghenem, Antonio Loria. Strict Lyapunov functions for time-varying systems with persistency of excitation. Automatica, 2017, 78, pp.274-279. 10.1016/j.automatica.2016.12.029 . hal01744598

\section{HAL Id: hal-01744598 \\ https://hal.science/hal-01744598}

Submitted on 5 Mar 2020

HAL is a multi-disciplinary open access archive for the deposit and dissemination of scientific research documents, whether they are published or not. The documents may come from teaching and research institutions in France or abroad, or from public or private research centers.
L'archive ouverte pluridisciplinaire HAL, est destinée au dépôt et à la diffusion de documents scientifiques de niveau recherche, publiés ou non, émanant des établissements d'enseignement et de recherche français ou étrangers, des laboratoires publics ou privés. 


\title{
Strict Lyapunov functions for time-varying systems with persistency of excitation
}

\author{
Mohamed Maghenem ${ }^{1} \quad$ Antonio Loría ${ }^{2}$ \\ M. Maghenem is with Univ. Paris Saclay. A. Loría is with CNRS. Address: Laboratoire des signaux et systèmes, 91192 \\ Gif-sur-Yvette,France.E-mail: mohamed.maghenem@lss.supelec.fr, antonio.loria@lss.supelec.fr
}

\begin{abstract}
We study the stability of the origin for a class of linear time-varying systems with a drift that may be divided in two parts. Under the action of the first, a function of the trajectories is guaranteed to converge to zero; under the action of the second, the solutions are restricted to a periodic orbit. Hence, by assumption, the system's trajectories are bounded. Our main results focus on two generic case studies that are motivated by common nonlinear control problems: model-reference adaptive control, control of nonholonomic systems, tracking control problems, to name a few. Then, based on the standing assumption that the system's dynamics is persistently excited, we construct a time-dependent Lyapunov function that has a negative definite derivative. Our main statements may be regarded as off-the-shelf tools of analysis for linear and nonlinear time-varying systems.
\end{abstract}

Key words: Linear time-varying systems, adaptive control, persistency of excitation.

\section{INTRODUCTION}

Lyapunov's first method is useful to approximate, locally, the nonlinear dynamics of a model by a linear differential equation. Similarly, if we are interested in establishing stability properties in the large (or even global), one may find it useful to rewrite a nonlinear ordinary differential equation as a linear time-varying system by replacing the states with the trajectories.

Regarding nonlinear systems as linear time-varying is not unusual. It is useful, for instance, in the analysis of adaptive control systems [10, pp. 626-627], in the stabilization of parameterized time-varying systems [25], in the analysis and design of observers for bilinear systems $[26,4]$, and in dynamic feedback stabilization, notably of chain-form systems [23].

Establishing uniform asymptotic stability of the origin for linear time-varying systems, however, is a difficult task in general. For instance, eigen-value analysis is generally inconclusive, even for boundedness of the solutions. A recurrent property found in the literature on analysis of time-varying systems is persistency of excitation, which was originally introduced in the context of systems identification [3] and it is known to be necessary and sufficient for uniform exponential stability of certain linear time-varying systems, see e.g. [19]. It also implies uniform global asymptotic stability for some nonlinear systems [22], notably in the context of model-reference adaptive control. For linear time-varying systems it is well-known that persistency of excitation is equivalent to the so-called uniform complete observability. Then, under this property and an output-injection argument, uniform global exponential stability follows [1].

For nonlinear time-varying systems, the notion of persistency excitation is intrinsically related to detectability $[2,11]$. However, since persistency excitation was introduced in the context of linear systems, as a property of functions that depend only on time, much of the literature on the subject does not apply directly to the analysis of nonlinear systems. Indeed, in general, the uniform character of asymptotic stability is lost [9], unless one explicitly assumes that persistency of excitation holds uniformly in the initial conditions $[25,14]$. In contrast to this, persistency of excitation is not necessary for weaker forms of stability such as bounded-input-bounded-state $[18,7]$.

Besides the question of stability lies that of stabilisation and, at a more fundamental level, that of controllability. A general definition of (non-uniform) asymptotic controllability, for nonlinear systems with time-varying parameters, is given for instance in [25]. Namely, it is established that under persistency of excitation of the input gain matrix (along the trajectories) one can always construct a control input to steer the trajectories to the origin with non-uniform rate of convergence. Systems with time-varying input gain appear naturally, e.g. in the stabilization of spacecrafts with magnetic actuators -see e.g., [24].

In stabilisation problems, persistency of excitation also 
appears naturally when there is a structural impediment to use autonomous smooth feedback, as in the case of chain-form systems [23]. In this reference, under a change of coordinates and a preliminary feedback, the closed-loop system is transformed into a so-called skewsymmetric system. Then, following the design rationale from [23], in [15] uniform global asymptotic stability was established for the closed-loop systems using controllers with persistency of excitation.

A common drawback of most works on persistency of excitation in the literature is that the methods of proof are based on tools such as Barbălat's lemma, properties of spaces of integrable functions, output-injection, etc., as opposed to Lyapunov's direct method. Yet the benefits of the latter cannot be overestimated. Having a strict Lyapunov function (with negative definite derivative) is an essential step in Lyapunov redesign to achieve robustness to unmodelled dynamics [21].

In this paper we present Lyapunov functions for certain linear time-varying systems with persistency of excitation. We broach two different case-studies motivated by scenarios reminiscent of model-reference adaptive control, stabilization of non-holonomic systems, and systems with time-varying input gain. The common thread between these case-studies is, as e.g. in [19], neutral stability; more precisely, we assume that we know a nonstrict Lyapunov function that ensures (non-asymptotic) stability. Our standing assumptions to guarantee attractivity are stated in terms of persistency of excitation. Our Lyapunov-function constructions are much inspired by $[16,17]$. However, to the best of our knowledge, for the classes of systems that we study, there does not exist equivalent results in the literature.

In next section we describe the class of systems that we study. Our main statements are presented in Section 3. Some concluding remarks are provided in Section 4.

\section{PROBLEM FORMULATION AND MOTI- VATION}

We address the question of stability, via Lyapunov's direct method, for a wide class of linear time-varying systems, defigned by

$$
\dot{x}=\left[\mathcal{A}_{o}(t)+\mathcal{A}_{s}(t)\right] x, \quad x \in \mathbb{R}^{n}
$$

where $\mathcal{A}_{o}$ and $\mathcal{A}_{s}$ are bounded differentiable mappings $\mathbb{R}_{\geq 0} \rightarrow \mathbb{R}^{n \times n}$.

The system (1) covers several systems from the literature. For instance, if $\mathcal{A}_{s}$ is constant and Hurwitz and $\mathcal{A}_{o}(t)$ is skew-symmetric we recover the class of systems studied in [19]. If, on the other hand, $\mathcal{A}_{o} \equiv 0$ and $\mathcal{A}_{s}(t)$ is negative semidefinite we recover the systems studied in [20]. A particular case of the latter are "gradient-type" adaptive systems, which are often written in the form $\dot{x}=-\Psi(t) \Psi(t)^{\top} x$, with $\Psi: \mathbb{R}_{\geq 0} \rightarrow \mathbb{R}^{m \times n}, m \geq n$. For such systems, with $\Psi$ bounded and with bounded derivative, it is well known that the origin is uniformly glob- ally exponentially stable if and only if $\Psi$ is persistently exciting [3,22], that is, if there exist $\mu>0$ and $T>0$ such that

$$
\int_{t}^{t+T} \Psi(s) \Psi(s)^{\top} d s \geq \mu I \quad \forall t \geq 0 .
$$

There are various proofs of this fact in the literature; see for instance [20,22], as well as [5,14] where tight stability bounds are also established.

Generally speaking, the model (1) has two essential constituting parts: the so-called oscillating drift $\mathcal{A}_{o}(t) x$ and the steering drift $\mathcal{A}_{s}(t) x$. Roughly speaking, under the action of the oscillating drift, the trajectories of (1) tend to oscillate while under the action of the steering drift there exists a function of the state and time, $(t, x) \mapsto h$ (e.g., part of the state), that vanishes. These properties are captured in the following hypothesis and they are illustrated by Example 1 farther below.

Assumption 1 1) There exist two bounded smooth functions $P_{s}$ and $Q_{s}$ taking values from $\mathbb{R}_{\geq 0}$ to $\mathbb{R}^{n \times n}$ such that, for all $t \geq 0, P_{s}(t)$ is symmetric positive definite, $Q_{s}(t)$ is symmetric positive semi-definite, $Q_{s}(t) \not \equiv 0$, and

$$
\mathcal{A}_{s}(t)^{\top} P_{s}(t)+P_{s}(t) \mathcal{A}_{s}(t)^{\top}+\dot{P}_{s}(t)=-Q_{s}(t) .
$$

2) There exists a smooth bounded function $P_{o}: \mathbb{R}_{\geq 0} \rightarrow$ $\mathbb{R}^{n \times n}$ such that, for all $t \geq 0, P_{o}(t)$ is symmetric positive definite and

$$
\mathcal{A}_{o}(t)^{\top} P_{o}(t)+P_{o}(t) \mathcal{A}_{o}(t)^{\top}+\dot{P}_{o}(t)=0
$$

Remark 1 In particular, the system $\dot{x}=\mathcal{A}_{o}(t) x$ with $\mathcal{A}_{o}$ satisfying item 2 ) above is called neutrally stable.

Example 1 (motivation) Let $a$ and $b$ be bounded persistently exciting functions and let

$$
\mathcal{A}_{s}(t):=\left[\begin{array}{cc}
-a(t) & 0 \\
0 & 0
\end{array}\right], \quad \mathcal{A}_{o}(t):=\left[\begin{array}{cc}
0 & -b(t) \\
b(t) & 0
\end{array}\right]
$$

Now, consider the system (1), (5) with $x=\left[x_{1}, x_{2}\right]^{\top} \in$ $\mathbb{R}^{2}$. This system satisfies Assumption 1 with $P_{s}=P_{o}=$ $I$. Hence, the derivative of $V(x)=x^{\top} P_{s} x=|x|^{2}$ along the trajectories of (1), (5) satisfies $\dot{V} \leq-a(t) x_{1}^{2} \leq 0$. Integrating the latter we obtain that $|\bar{x}(t)|$ is bounded for all $t \geq 0$ and $\sqrt{a} x_{1} \in \mathcal{L}_{2}$. Therefore, using standard arguments based on Barbălat's lemma, we may conclude that $h(t, x)=\sqrt{a(t)} x_{1}$ converges to zero [10].

Now, for the sake of argument, let us regard $h(t, x)=$ $\sqrt{a(t)} x_{1}$ as a converging output. Then, $x_{2}$ also converges if the system is observable with respect to $h$, which holds if and only if $b$ is persistently exciting [1]. For general classes of time-varying systems, the weaker property of 
detectability has also been linked to persistency of excitation - see [11].

For the system defined by (1) and (5), however, persistency of excitation of $b$ does not suffice, in general, to establish the convergence of the state trajectories because $a$ varies with time too. For instance, let $a$ and $b$ be piecewise constant periodic functions taking non-negative values, hence persistently exciting. More specifically, for each integer $n \geq 0$, let $J_{n}:=(\pi / \bar{b})[2 n+1,2 n+2]$ and let $\mathcal{J}:=\cup_{n>0} J_{n}$. Then, let $\bar{a}, \bar{b}>0$ and, for all $t \in \mathcal{J}$, let $a(t):=0$ and $b(t):=\bar{b}$ while, for all $t \notin \mathcal{J}$ let $a(t):=\bar{a}$ and $b(t):=0$. Hence, $a(t) b(t) \equiv 0$. For this particular choice of $a$ and $b$, the trajectories $x_{1}(t)$ and $x_{2}(t)$ generated by (1), (5), with $\bar{a}=\bar{b}=1$ and initial condition $t_{\circ}=0, x_{1}(0)=0$, and $x_{2}(0)=1$, do not converge to zero, but oscillate.

\section{MAIN RESULTS}

Our main statements apply to systems of the form (1) under the standing Assumption 1. We focus our attention on two case-studies that include several problems in the realm of adaptive control, tracking control, and stabilization of nonholonomic systems, to name a few. For both cases, we give conditions for uniform global exponential stability and, more significantly, we establish our results by providing original strict Lyapunov functions.

The construction method for all our Lyapunov functions is inspired from [16,17]. It relies on a functional that is defined upon a locally Lipschitz bounded function $\psi$ : $\mathbb{R}_{\geq 0} \rightarrow \mathbb{R}$ with bounded first derivative (a.e.), that is, we assume that there exists $\bar{\psi}>0$, such that

$$
\max \left\{|\psi|_{\infty},|\dot{\psi}|_{\infty}\right\} \leq \bar{\psi} \quad \text { a.e. }
$$

where

$$
|\psi|_{\infty}:=\operatorname{ess} \sup _{t \geq 0}|\psi(t)|
$$

and, depending on the context, we use $|\cdot|$ to denote the absolute value of scalars, the Euclidean norm of vectors, or the induced $\mathcal{L}_{2}$ norm of matrices.

In addition, we assume that $\psi$ is persistently exciting. Then, we define

$$
\Upsilon_{\psi}(t):=1+2 \bar{\psi}^{2} T-\frac{2}{T} \int_{t}^{t+T} \int_{t}^{m} \psi(s)^{2} d s d m
$$

and, for further development, we remark that

$$
1 \leq \Upsilon_{\psi}(t)<1+2 \bar{\psi}^{2} T
$$

Furthermore, after the fundamental theorem of calculus, we have

$$
\dot{\Upsilon}_{\psi}(t)=-\frac{2}{T} \int_{t}^{t+T} \psi(s)^{2} d s+2 \psi(t)^{2}
$$

hence, since $\psi$ is persistently exciting,

$$
\dot{\Upsilon}_{\psi}(t) \leq-\frac{2 \mu}{T}+2 \psi(t)^{2}
$$

\subsection{First-case study: "adaptive-control" systems}

Our first case-study is the system

$$
\begin{cases}\dot{x}_{1}=A(t) x_{1}-B(t) x_{2}, & x_{1} \in \mathbb{R}^{n} \\ \dot{x}_{2}=C(t) x_{1}, & x_{2} \in \mathbb{R}^{m}\end{cases}
$$

where $A, B$ and $C$ are assumed to be piece-wise continuous and bounded function mapping $\mathbb{R}_{\geq 0}$ into Euclidean spaces of appropriate dimensions. The equations (11) may be written in the compact form (1) with $x:=$ $\left[x_{1}, x_{2}\right]^{\top}$,

$$
\mathcal{A}_{s}=\left[\begin{array}{cc}
A(t) & 0 \\
0 & 0
\end{array}\right], \quad \mathcal{A}_{o}(t)=\left[\begin{array}{cc}
0 & -B(t) \\
C(t) & 0
\end{array}\right]
$$

and cover, in addition to Example 1, a familiar system studied in the context of model-reference adaptive control $[22,10,8]$. In such case, $A$ is assumed to be constant (hence, also $\mathcal{A}_{s}$ ) and Hurwitz, that is, there exists $P=P^{\top}>0$ such that $A^{\top} P+P A=:-Q<0$. Moreover, if in addition we have $C(t):=B(t)^{\top} P$, we see that the derivative of $V(x)=x_{1}^{\top} P x_{1}+\left|x_{2}\right|^{2}$ along the trajectories of (1), (12) yields $\dot{V}(x) \leq-x_{1}^{\top} Q x_{1}$. Then, as for Example 1 (with $a$ constant), using standard arguments one may conclude that $x_{1} \rightarrow 0$ and the solutions are bounded $[22,10,8]$. Furthermore, there are numerous (rather lengthy) proofs of the fact that the origin is uniformly exponentially stable if, moreover, $B$ is persistently exciting; see some of the above-cited references.

As Example 1 also illustrates, however, the problem is much more complex if $\mathcal{A}_{s}$ is not constant. In that regard, we must mention [17] where a statement on uniform global asymptotic stability (UGAS) for systems with a structure similar to (1), (12) (with $C=B$ ) is given. In this reference $B$ and $C$ depend both on time and the state and $A$ is a nonlinear function of $x_{1}$ satisfying $x_{1}^{\top} A\left(x_{1}\right) x_{1} \geq c\left|x_{1}\right|^{2}$ for some $c>0$. The first straightforward proof of UGAS for model-reference adaptive control systems, via Lyapunov's direct method, was provided.

Our first result provides, for the first time, a proof of exponential stability for systems (1), (12) under Assumption 1 and, moreover, we provide a strict Lyapunov function. In particular, we relax the uniform-positivity condition on $A$ made in [17].

Theorem 2 For the system (11) assume that $B \in \mathcal{C}^{1}$. In addition, assume that there exist once-continuouslydifferentiable functions $P: \mathbb{R}_{\geq 0} \rightarrow \mathbb{R}_{>0}^{n \times n}$ and $Q: \mathbb{R}_{\geq 0} \rightarrow$ 
$\mathbb{R}_{\geq 0}^{n \times n}$, as well as positive constants $P_{m}$ and $P_{M}$, such that

$$
\begin{gathered}
P_{m} I \leq P(t) \leq P_{M} I \\
\dot{P}(t)+A(t)^{\top} P(t)+P(t) A(t) \leq-Q(t) \leq 0 \\
C(t)=B(t)^{\top} P(t) .
\end{gathered}
$$

Finally, assume that the function $\psi: \mathbb{R}_{\geq 0} \rightarrow \mathbb{R}_{\geq 0}$ defined by

$$
\psi(t):=\lambda_{m}(Q(t)) \sqrt{\lambda_{m}\left(B(t)^{\top} B(t)\right)},
$$

where $\lambda_{m}$ denotes the smallest eigenvalue, is persistently exciting and satisfies (6). Then, the null solution of (11) is uniformly exponentially stable and the system admits the strict Lyapunov function

$$
\begin{aligned}
V(t, x)=\lambda_{m}^{2}(Q(t)) x_{1}^{\top} B(t) x_{2} \\
+\frac{1}{2}\left[\Upsilon_{\psi}(t)+\alpha\right]\left[x_{1}^{\top} P(t) x_{1}+\left|x_{2}\right|^{2}\right]
\end{aligned}
$$

with $\psi$ as in (14) and ${ }^{1}$

$$
\begin{aligned}
\alpha & \geq(2 T / \mu) \lambda_{m}^{3}(Q)|\dot{B}|_{\infty}^{2}+(8 T / \mu) \lambda_{m}(Q) \dot{\lambda}_{m}^{2}(Q)|B|_{\infty}^{2} \\
& +(2 T / \mu) \lambda_{m}^{3}(Q)\left|A^{\top} B\right|_{\infty}^{2}+\lambda_{m}^{2}(Q)|B|_{\infty}^{2} / P_{m} \\
& +2 \lambda_{m}(Q) \lambda_{m}\left(B^{\top} B\right) P_{M}+2 \lambda_{m}(Q)|B C|_{\infty} .
\end{aligned}
$$

Indeed, we have $\dot{V}(t, x) \leq-(\mu / 4 T)\left[x_{1}^{\top} P x_{1}+\left|x_{2}\right|^{2}\right]$.

Proof. In view of (8), the boundedness of $B, Q$, and $P$, as well as (15), $V$ is positive definite and radially unbounded. Indeed, there exist $\eta_{1}, \eta_{2}>0$ such that

$$
\eta_{1}|x|^{2} \leq V(t, x) \leq \eta_{2}|x|^{2}, \quad x=\left[x_{1}^{\top} x_{2}^{\top}\right]^{\top} .
$$

The time derivative of $V$ along trajectories of (11) satisfies

$$
\begin{aligned}
\dot{V}(t, x) \leq & 2 \lambda_{m}(Q) \dot{\lambda}_{m}(Q) x_{1}^{\top} B x_{2}+\lambda_{m}^{2}(Q) x_{1}^{\top} A^{\top} B x_{2} \\
& -\lambda_{m}^{2}(Q) x_{2}^{\top} B^{\top} B x_{2}+\lambda_{m}^{2}(Q) x_{1}^{\top} B C x_{1} \\
& +\lambda_{m}^{2}(Q) x_{1}^{\top} \dot{B} x_{2}-\frac{\alpha}{2} x_{1}^{\top} Q x_{1} \\
& -\frac{\mu}{T}\left[x_{1}^{\top} P x_{1}+\left|x_{2}\right|^{2}\right]+\psi^{2}\left[x_{1}^{\top} P x_{1}+\left|x_{2}\right|^{2}\right] \\
\leq & -\frac{\alpha}{2} \lambda_{m}(Q)\left|x_{1}\right|^{2}+\lambda_{m}^{2}(Q) \lambda_{m}\left(B^{\top} B\right) x_{1}^{\top} P x_{1} \\
& +\lambda_{m}^{2}(Q) x_{1}^{\top} B C x_{1}+\lambda_{m}^{2}(Q) x_{1}^{\top} A^{\top} B x_{2} \\
+ & 2 \lambda_{m}(Q) \dot{\lambda}_{m}(Q) x_{1}^{\top} B x_{2}+\lambda_{m}^{2}(Q) x_{1}^{\top} \dot{B} x_{2} \\
- & \frac{\mu}{T}\left|x_{2}\right|^{2}-\frac{\mu}{T} x_{1}^{\top} P x_{1} .
\end{aligned}
$$

Then, we use the inequalities

$$
\lambda_{m}^{2}(Q) x_{1}^{\top} \dot{B} x_{2} \leq \frac{\epsilon}{2} \lambda_{m}^{4}(Q)|\dot{B}|_{\infty}^{2}\left|x_{1}\right|^{2}+\frac{1}{2 \epsilon}\left|x_{2}\right|^{2},
$$

\footnotetext{
1 We drop the argument $(t)$ from $Q$ and $B$, not to make the notation even more cumbersome.
}

$$
\begin{aligned}
& 2 \lambda_{m}(Q) \dot{\lambda}_{m}(Q) x_{1}^{\top} B x_{2} \leq \\
& 2 \epsilon \lambda_{m}^{2}(Q) \dot{\lambda}_{m}^{2}(Q)|B|_{\infty}^{2}\left|x_{1}\right|^{2}+\frac{1}{2 \epsilon}\left|x_{2}\right|^{2}, \\
& \lambda_{m}^{2}(Q) x_{1}^{\top} A^{\top} B x_{2} \leq \frac{\epsilon}{2} \lambda_{m}^{4}(Q)\left|A^{\top} B\right|_{\infty}^{2}\left|x_{1}\right|^{2}+\frac{1}{2 \epsilon}\left|x_{2}\right|^{2},
\end{aligned}
$$

which hold for any $\epsilon>0$. Hence, setting $\epsilon=2 T / \mu$ and in view of $(15)$, it follows that $\dot{V} \leq-(\mu / 4 T)\left|x_{2}\right|^{2}-$ $(\mu / T) x_{1}^{\top} P x_{1}$.

In the case that $x_{1}, x_{2} \in \mathbb{R}$ we obtain the following.

Corollary 1 Let $\bar{a}, \bar{b}>0$. Consider the system (1), (5) with $a(t), b(t) \geq 0$ for all $t \geq 0$ and such that, for almost all $t \geq 0$,

$$
\max \left\{|a|_{\infty},|\dot{a}|_{\infty}\right\} \leq \bar{a}, \quad \max \left\{|b|_{\infty},|\dot{b}|_{\infty}\right\} \leq \bar{b}
$$

Assume, in addition, that $\psi:=a b$ is persistently exciting. Then, the function $V: \mathbb{R}_{\geq 0} \times \mathbb{R}^{2} \rightarrow \mathbb{R}_{\geq 0}$, defined as

$$
V(t, x)=a(t)^{2} b(t) x_{1} x_{2}+\frac{1}{2}\left[\Upsilon_{a b}(t)+\alpha\right]|x|^{2},
$$

with

$$
\alpha \geq \bar{a} \bar{b}^{2}\left[4+\bar{a}+\frac{2 \bar{a}^{2} T}{\mu}\left(5+\bar{a}^{2}\right)\right],
$$

satisfies $\dot{V}(t, x) \leq-(\mu / 4 T)|x|^{2}$.

Example 2 Consider the master-slave synchronization problem for two harmonic oscillators, the slave system $\dot{z}=\mathcal{A}_{o}(t) z+B u$ and the master $\dot{z}^{*}=\mathcal{A}_{o}(t) z^{*}$, where

$$
\mathcal{A}_{o}(t):=\left[\begin{array}{cc}
0 & -\omega(t) \\
\omega(t) & 0
\end{array}\right], \quad B=\left[\begin{array}{l}
1 \\
0
\end{array}\right]
$$

and $z:=\left[\begin{array}{ll}z_{1} & z_{2}\end{array}\right]^{\top}$. That is, both oscillators spin at the same variable frequency $\omega(t)$, but out of phase. Then, the problem consists in ensuring that $z$ converges to $z^{*}$ exponentially fast under the assumption that the oscillators are linked through an unreliable channel.

To model the channel unreliability, we introduce the function $a: \mathbb{R}_{\geq 0} \rightarrow \mathbb{R}_{\geq 0}$ which is assumed to be bounded and equal to zero over certain intervals of time of length smaller than a threshold. For instance, $a(t)$ may be a piece-wise constant function as in Example 1. Then, we design a simple control law so that the closed-loop system have the structure (1): a steering drift and an oscillating drift. The latter is natural to the harmonic oscillators while the former may be added through the simple time-varying output feedback $u=-a(t)\left[z_{1}-z_{1}^{*}\right]$. Then, the closed-loop system has exactly the form (1), (5) with $x_{1}:=z_{1}-z_{1}^{*}$ and $b(t):=\omega(t)$. We conclude that phase-lock synchronisation is achieved provided that $a$ and $\omega$ are bounded, have bounded derivatives, and their product is persistently exciting. Moreover, Corollary 1 provides a Lyapunov function. 


\subsection{Second case-study: skew-symmetric systems.-}

We address a second generic case-study which is motivated by stabilization problems in which nonautonomous feedback is imposed by the control problem. These include: leader-follower tracking control [12], stabilization of non-holonomic systems [23,15], stabilization of systems with time-varying input gain [13,24], to name a few.

We consider systems defined by the ordinary differential equation (1) with

$$
\mathcal{A}_{s}(t):=-b(t)^{2} B B^{\top}, \quad \mathcal{A}_{o}(t):=a(t) A,
$$

where $A \in \mathbb{R}^{n \times n}, B \in \mathbb{R}^{n \times m}$ and, in addition, we assume the following:

1) the pair $(A, B)$ is controllable,

2) $A$ is neutrally stable (e.g., skew-symmetric), and

3) both $t \mapsto a$ and $t \mapsto b$ are scalar continuously differentiable functions such that the function $\psi:=a^{2} b$ is persistently exciting.

The class of systems modelled by (1), (19) intersects with the systems studied in [19] and covers the systems studied in [6], where uniform global exponential stability is ensured for the particular case that $\mathcal{A}_{o}$ is constant and skew-symmetric. More significantly, in the latter reference the proof relies on trajectory-based arguments, whereas in this paper we give a strict Lyapunov function.

Theorem 3 Consider the system (1), (19) where $t \mapsto a$, $t \mapsto b$ satisfy (17) for almost all $t \geq 0$ and such that $\psi:=a^{2} b$ is persistently exciting. Assume, in addition, that the pair $(A, B)$ is controllable and that there exist a positive definite matrix $P=P^{\top} \in \mathbb{R}^{n \times n}$, and $C \in \mathbb{R}^{n \times n}$ such that

$$
\begin{aligned}
& A^{\top} P+P A=0, \\
& P B B^{\top}=B B^{\top} P=: C C^{\top},
\end{aligned}
$$

and (13) hold. Define $V: \mathbb{R}_{\geq 0} \times \mathbb{R}^{n} \rightarrow \mathbb{R}_{\geq 0}$ as

$$
\begin{aligned}
V(t, x):=\frac{1}{2}\left[\gamma+\Upsilon_{a^{2} b}(t)\right] x^{\top} P x \\
\\
\quad+b(t)^{2} a(t)^{3} x^{\top} P A \sum_{i=1}^{n} \beta_{i} \Gamma_{i} P x
\end{aligned}
$$

where $\gamma:=\gamma_{1}+\gamma_{2}$,

$$
\begin{aligned}
\gamma_{1} & :=\frac{T \bar{b}^{6} \bar{a}^{6}}{2 \mu P_{m}}\left|\sum_{i=1}^{n} \beta_{i} C^{\top}\left[A \Gamma_{i} P-\Gamma_{i} P A\right]\right|^{2} \\
\gamma_{2} & :=\frac{T \bar{a}^{4}}{\mu P_{m}} \sum_{i=1}^{n} \beta_{i}\left|M^{\top} P C\right|_{\infty}^{2}+\frac{2 \bar{b}^{2} \bar{a}^{3}}{P_{m}}\left|P A \sum_{i=1}^{n} \beta_{i} \Gamma_{i} P\right|
\end{aligned}
$$$$
\Gamma_{i}:=\sum_{j=1}^{i} A^{j-1} B B^{\top} A^{j-1 \top}
$$

$\beta_{n} I \geq\left[P A \Gamma_{n} A^{\top}\right]^{-1}$ and $\beta_{i}$ is defined in reverse order, i.e., for each $i \in\{n-1, \cdots, 1\}$,

$$
\beta_{i} \geq \frac{2 n T}{\mu P_{m}}\left|\left[P A^{i} B\right]^{\top} M\right|_{\infty}^{2}\left[\sum_{k=i+1}^{n} \beta_{k}\right]^{2}-\sum_{k=i+1}^{n-1} \beta_{k}
$$

where $M=[2 \dot{b} a+3 b \dot{a}] A+b a^{2} A^{2}$. Then, $V$ is a strict differentiable Lyapunov function for (1), (19) and the origin is uniformly globally exponentially stable.

Remark 4 In view of conditions (20) and (13), Assumption 1 holds.

Proof. In view of (8), the boundedness of $a$ and $b$, and the bound $\gamma>\gamma_{2}$, the function $V$ is positive definite and radially unbounded. Indeed, there exist $\eta_{1}, \eta_{2}>0$ such that

$$
\eta_{1}|x|^{2} \leq V(t, x) \leq \eta_{2}|x|^{2}
$$

for all $t \geq 0$ and $x \in \mathbb{R}^{n}$.

Next, we compute the total derivative of $V$ in (22) along the trajectories of (1), (19). For this, we use (10), (20), and the persistency of excitation of the product $\psi=a^{2} b$. Then, we reorganise some terms to obtain

$$
\begin{aligned}
& \dot{V} \leq-\gamma b^{2} x^{\top} C C^{\top} x-\frac{\mu}{T} x^{\top} P x \\
& -a^{4} b^{2} x^{\top}\left[\beta_{n} P A \Gamma_{n} A^{\top} P-P+\sum_{i=1}^{n-1} \beta_{i} P A \Gamma_{i} A^{\top} P\right] x \\
& -b a^{2}\left[[2 \dot{b} a+3 b \dot{a}][P A x]^{\top}+b a^{2}\left[P A^{2} x\right]^{\top}\right] \sum_{i=1}^{n} \beta_{i} \Gamma_{i} P x \\
& -b^{4} a^{3}\left[C^{\top} x\right]^{\top} \sum_{i=1}^{n} \beta_{i} C^{\top}\left[A \Gamma_{i} P-\Gamma_{i} P A\right] x .
\end{aligned}
$$

To establish that $\dot{V}$ is negative definite we first note that, since the pair $A, B$ is controllable by assumption, the matrix $\Phi_{c} \Phi_{c}^{\top}$ where $\Phi_{c}$ corresponds to Kalman's controllability matrix $\Phi_{c}:=\left[B A B \cdots A^{n-1} B\right]$, is positive definite. Note, moreover, that $\Gamma_{n}=\Phi_{c} \Phi_{c}^{\top}$ hence, in view of the definition of $\beta_{n}$, we have $-\beta_{n} x^{\top} P A \Gamma_{n} A^{\top} P x+$ $x^{\top} P x \leq 0$. Therefore, the sum of the first two terms in the second line of (27) is non-positive. Next, note that the terms in the last line of (27) are bounded from above by

$$
\gamma_{1} b^{2}\left|C^{\top} x\right|^{2}+\frac{\mu P_{m}}{2 T}|x|^{2}
$$

where $\gamma_{1}$ is defined in (22). Hence, using $\Gamma_{1}=B B^{\top}$ and $\Gamma_{i}=B B^{\top}+A \Gamma_{i-1} A^{\top}$ for all $i \geq 2$, as well as (20b), it follows that

$$
\begin{gathered}
\dot{V} \leq-b a^{2} x^{\top} M^{\top}\left[P \sum_{i=1}^{n} \beta_{i} C C^{\top}+\sum_{i=2}^{n} \beta_{i} P A \Gamma_{i-1} A^{\top} P\right] x \\
-\gamma_{2} b^{2}\left|C^{\top} x\right|^{2}-\frac{\mu}{2 T} x^{\top} P x-b^{2} a^{4} x^{\top} \sum_{i=1}^{n-1} \beta_{i} P A \Gamma_{i} A^{\top} P x
\end{gathered}
$$


where $M$ is defined below (25). Next, observe that

$$
\begin{aligned}
\sum_{i=2}^{n} \beta_{i} P A \Gamma_{i-1} A^{\top} P & =\sum_{i=2}^{n} \beta_{i} \sum_{j=1}^{i-1} P A^{j} B B^{\top} A^{j \top} P \\
& =\sum_{j=1}^{n-1} P A^{j} B B^{\top} A^{j \top} P \sum_{i=j+1}^{n} \beta_{i} \\
\sum_{i=1}^{n-1} \beta_{i} P A \Gamma_{i} A^{\top} P & =\sum_{i=1}^{n-1} \beta_{i} \sum_{j=1}^{i} P A^{j} B B^{\top} A^{j \top} P \\
& =\sum_{j=1}^{n-1} P A^{j} B B^{\top} A^{j \top} P \sum_{i=j}^{n-1} \beta_{i}
\end{aligned}
$$

so, in view of (23), we obtain

$$
\begin{gathered}
\dot{V} \leq-\frac{\mu}{4 T} x^{\top} P x-b^{2} a^{4} x^{\top}\left[\sum_{j=1}^{n-1} P A^{j} B B^{\top} A^{j \top} P \sum_{i=j}^{n-1} \beta_{i}\right] x \\
-b a^{2} x{ }^{\top} M^{\top}\left[\sum_{j=1}^{n-1} P A^{j} B B^{\top} A^{j \top} P \sum_{i=j+1}^{n} \beta_{i}\right] x
\end{gathered}
$$

Then, defining $Y_{j}:=\left[P A^{j} B\right]^{\top}$, it follows that

$$
\begin{aligned}
\dot{V} \leq-\frac{\mu}{4 T} x^{\top} P x-\sum_{j=1}^{n-1}\left[\left|b a^{2} Y_{j} x\right|^{2} \sum_{i=j}^{n-1} \beta_{i}\right. \\
\left.+\left[Y_{j} M x\right]^{\top}\left[b a^{2} Y_{j} x\right] \sum_{i=j+1}^{n} \beta_{i}\right] .
\end{aligned}
$$

Using the triangle inequality on the last term on the right hand side of (29), we see that for any $\epsilon_{j} \neq 0$,

$$
\begin{aligned}
\dot{V} & \leq-\frac{\mu}{4 T} x^{\top} P x+\frac{1}{2} \sum_{j=1}^{n-1} \epsilon_{j}\left|Y_{j} M x\right|^{2} \\
& -\sum_{j=1}^{n-1}\left|b a^{2} Y_{j} x\right|^{2}\left[\left[\beta_{j}+\sum_{i=j+1}^{n-1} \beta_{i}\right]-\frac{1}{2 \epsilon_{j}}\left[\sum_{i=j+1}^{n} \beta_{i}\right]^{2}\right] .
\end{aligned}
$$

Now, on one hand, defining

$$
\epsilon_{j}=\frac{\mu P_{m}}{4 n T\left|Y_{j} M\right|_{\infty}^{2}}
$$

we obtain

$$
-\frac{\mu}{8 T} x^{\top} P x+\frac{1}{2} \sum_{j=1}^{n-1} \epsilon_{j}\left|Y_{j} M x\right|^{2} \leq 0 .
$$

On the other, in view of (25), we have

$$
\beta_{j}+\sum_{i=j+1}^{n-1} \beta_{i} \geq \frac{1}{2 \epsilon_{j}}\left(\sum_{i=j+1}^{n} \beta_{i}\right)^{2} .
$$

Thus, we conclude that

$$
\dot{V} \leq-\frac{\mu}{8 T} x^{\top} P x
$$

which completes the proof.

We wrap up this section with two concrete examples of control of physical systems in which our statement for systems of the form (1), (19) provides a clear improvement on the literature. The first concerns the so-called skew-symmetric systems. This terminology was coined in [23] in the context of stabilization of under-actuated nonholonomic systems in chain form. Skew-symmetric systems are also studied in the context of stability analysis, in [14] and, in turn, the results therein were used in [15] to construct smooth controllers for nonholonomic systems.

Example 3 [Skew-symmetric systems] Roughly speaking, the systems studied in $[23,14,15]$ have the form

$$
\dot{x}=\left[\begin{array}{ccccc}
-k_{1} & -k_{2} a(t) & 0 & \cdots & 0 \\
a(t) & 0 & -k_{3} a(t) & 0 & \vdots \\
0 & a(t) & 0 & \ddots & 0 \\
\vdots & 0 & \ddots & \ddots & -k_{n} a(t) \\
0 & \cdots & 0 & a(t) & 0
\end{array}\right] x
$$

where $k_{i}>0$ and $a$ is persistently exciting, bounded and with bounded derivative. This system may be written in the compact (1), (19) with $b(t) \equiv \sqrt{k_{1}}, B=[1,0 \cdots 0]$ hence, with $\mathcal{A}_{s}=\operatorname{diag}\left[\begin{array}{cccc}-k_{1} & 0 & \cdots & 0\end{array}\right]$, and an obvious choice for $\mathcal{A}_{o}(t)$.

We stress that even though the resulting $\mathcal{A}_{o}(t)$ is not skew-symmetric, it is neutrally stable. That is, it satisfies (4) with $P_{o}:=\operatorname{diag}\left[1, k_{2}, k_{2} k_{3}, \cdots, \prod_{i=2}^{m-1} k_{i}\right]$. Moreover, (3) holds with $P_{s}=P_{o}$ and $Q_{s}=-\mathcal{A}_{s}$. Hence, Assumption 1 holds. Besides, it takes a short calculation to verify that the conditions of Theorem 3 hold.

Our second example concerns tracking control of underactuated ships.

Example 4 (After [12]). In this reference a tracking control problem for underactuate ships was solved under the assumption that the reference trajectories are persistently exciting. Interestingly, the closed-loop system in this reference has the cascaded form

$$
\begin{array}{ll}
\dot{x}_{1}=\left[\mathcal{A}_{s}+\mathcal{A}_{o}(t)\right] x_{1}+G\left(t, x_{1}, x_{2}\right) x_{2}, & x_{1} \in \mathbb{R}^{4} \\
\dot{x}_{2}=F x_{2}, & x_{2} \in \mathbb{R}^{2}
\end{array}
$$


where $F$ is a Hurwitz constant matrix, $\mathcal{A}_{s}$ is diagonal constant with two negative elements and two zero elements, $G$ has linear growth in $x_{1}$ and $\mathcal{A}_{o}$ depends on reference trajectories and satisfies item 2 of Assumption 1 -see [12] for details. Following standard arguments for cascaded systems it is possible to establish uniform global asymptotic stability of the origin, provided that the same property holds for the nominal system $\dot{x}_{1}=\left[\mathcal{A}_{s}+\mathcal{A}_{o}(t)\right] x_{1}$. In [12] this is established under persistency of excitation of the reference trajectories along with uniform-complete-observability and outputinjection arguments. Theorem 3 not only delivers a strict Lyapunov function to ensure exponential stability for the nominal $x_{1}$-dynamics but it also constitutes a fundamental step to carry on a robustness analysis with respect to unmodelled perturbations.

\section{CONCLUSIONS}

We have presented original strict Lyapunov functions for linear time-varying systems with persistency of excitation and established several statements of uniform exponential stability. These results may be applied in the analysis of nonlinear systems provided that the appropriate assumptions are imposed to guarantee uniformity. In that regard, we believe that the construction of strict Lyapunov functions for nonlinear time-varying systems with a similar structure as that investigated here (see Assumption 1) is an interesting open problem. Notably, the problem of establishing robustness properties (Input-tooutput stability) is a well-motivated avenue of research for which our statements may be a starting point.

\section{References}

[1] B. D. O. Anderson, R.R. Bitmead, C.R. Johnson, Jr., P.V. Kokotović, R.L. Kosut, I. Mareels, L. Praly, and B.D. Riedle. Stability of adaptive systems. The MIT Press, Cambridge, MA, USA, 1986.

[2] Z. Artstein. Stability, observability and invariance. J. Diff. Eqs., 44:224-248, 1982.

[3] K. J. Åstrom and Bohn. Numerical identification of linear dynamic systems from normal operating records. In P. H. Hammond, editor, Proc. of the 2nd IFAC Symp. on Theory of Self-adaptive Control Systems, pages 96-111, Nat. Phys. Lab., Teddington, England, 1965.

[4] G. Besançon. A viewpoint on observability and observer design for nonlinear systems, chapter in New directions in nonlinear observer design. Lecture Notes in Control and Information Sciences. Springer Verlag, H. Nijemeijer, T. I. Fossen, eds., London, 1999.

[5] R. Brockett. The rate of descent for degenerate gradient flows. In Proc. Math. Theory of Networks and Systems, 2000.

[6] A. Chaillet, Y. Chitour, A. Loría, and M. Sigalotti. Uniform stabilization for linear systems with persistency of excitation. The neutrally stable and the double integrator cases. Math. of Cont. Sign. and Syst., 20(2):136-156, 2008.

[7] G. C. Goodwin and E. K. Teoh. Adaptive control of a class of linear time-varying systems. In IFAC Workshop on Adaptive Systems in Control and Signals Processing, June 1983.
[8] P. Ioannou and J. Sun. Robust adaptive control. Prentice Hall, New Jersey, USA, 1996.

[9] I. Karafyllis and J. Tsinias. Non-uniform in time stabilization of linear systems and tracking control of non-holonomic systems. Int. J. of Contr., 76(15):1536-1546, 2003.

[10] H. Khalil. Nonlinear systems. Macmillan Publishing Co., 2nd ed., New York, 1996.

[11] T. C. Lee. On the equivalence relations of detectability and PE conditions with applications to stability analysis of time-varying systemss. In Proc. IEEE American Control Conference, June 2003.

[12] E. Lefeber, K. Y. Pettersen, and H. Nijmeijer. Tracking control of an underactuated ship. IEEE Trans. Contr. Syst. Technol., 11(1):52-61, 2003.

[13] A. Loría, A. Chaillet, G. Besançon, and Y. Chitour. On the $\mathrm{PE}$ stabilization of time-varying systems: open questions and preliminary answers. In Proc. 44th. IEEE Conf. Decision. Contr, pages 6847-6842, 2005.

[14] A. Loría and E. Panteley. Uniform exponential stability of linear time-varying systems: revisited. Syst. $\&$ Contr. Letters, 47(1):13-24, 2002.

[15] A. Loría, E. Panteley, and K. Melhem. UGAS of skewsymmetric time-varying systems: application to stabilization of chained form systems. European J. of Contr., 8(1):33-43, 2002.

[16] M. Malisoff and F. Mazenc. Constructions of Strict Lyapunov functions. Springer Verlag, London, 2009.

[17] F. Mazenc, M. de Queiroz, and M. Malisoff. Uniform global asymptotic stability of a class of adaptively controlled nonlinear systems. IEEE Trans. on Automat. Contr., 54(5):1152-1158, 2009.

[18] R. H. Middleton and G. Goodwin. Adaptive control of timevarying linear systems. IEEE Trans. on Automat. Contr., 33(2):150-155, 1988.

[19] A. P. Morgan and K. S. Narendra. On the stability of nonautonomous differential equations $\dot{x}=[A+B(t)] x$ with skew-symmetric matrix $B(t)$. SIAM J. on Contr. and Opt., 15(1):163-176, 1977.

[20] A. P. Morgan and K. S. Narendra. On the uniform asymptotic stability of certain linear nonautonomous differential equations. SIAM J. on Contr. and Opt., 15(1):524, 1977.

[21] K. S. Narendra and A. M. Annaswamy. A new adaptive law for robust adaptation without persistent excitation. IEEE Trans. on Automat. Contr., 32(2):134-145, 1987.

[22] K. S. Narendra and A. M. Annaswamy. Stable adaptive systems. Prentice-Hall, Inc., New Jersey, 1989.

[23] C. Samson. Control of chained system: Application to path following and time-varying point stabilization of mobile robots. IEEE Trans. on Automat. Contr., 40(1):64-77, 1995.

[24] S. Sukumar and M. R. Akella. Precision attitude stabilization: Incorporating rise and fall times in gas-based thrusters. J. Guid. Contr. dyn., 34(1):317-323, 2011.

[25] J. Tsinias. Links between asymptotic controllability and persistence of excitation for a class of time-varying systems. Syst. \& Contr. Letters, 54:1051-1062, 2005.

[26] Q. Zhang. Adaptive observers for MIMO linear time-varying systems. IEEE Trans. on Automat. Contr., 47(3):525-529, 2002. 